CASE REPORT

R.R. Watson

C. Russo

\section{Upper Extremity Arteriovenous Dialysis Fistula Resulting in Cavernous Sinus Arterialized Blood Flow}

SUMMARY: Arterialized blood flow in the cavernous sinus may result from carotid-cavernous fistula or dural venous fistula. We encountered an unusual case of arterialized blood flow in the cavernous sinus on MR angiography resulting from arterialized retrograde venous flow in the internal jugular vein. This abnormal flow originated from an upper extremity dialysis arteriovenous fistula in the presence of central venous occlusion. The patient's symptoms of visual disturbance resolved after the central venous occlusion was treated with stent placement.
A 36-year-old woman with a history of end stage renal disease, dialysis dependence, and left-arm arteriovenous (AV) fistula was hospitalized with vague abdominal pain and possible bowel obstruction. After 7 days in the hospital, she reported blurriness of vision and eventually provided a history of intermittent visual blurriness and headaches during the past few weeks, as well as mild left-arm pain.

Noncontrast MR imaging of the head revealed prominence of the left superior ophthalmic vein and mild left proptosis. Intracranial time-of-flight MR angiography (MRA) was also performed (Fig 1A). This showed abnormally increased signal intensity within the left cavernous sinus, the left transverse sinus, the left inferior petrosal sinus, and the ipsilateral superior ophthalmic vein. This signal intensity was interpreted as suggestive of carotid cavernous sinus fistula or dural venous fistula, and a catheter angiogram was recommended.

A conventional cerebral angiogram was then performed. There was no evidence of carotid-cavernous fistula (CCF) (Fig 1B). Injection of the bilateral internal carotid, selective bilateral external carotid, and bilateral vertebral arteries all showed no evidence of dural venous fistula. Contrast injection of the aortic arch revealed normal great vessels, but the late arterial phase showed early flow into the left subclavian vein, as expected with AV fistula. Venous phase images showed that the left brachiocephalic vein was occluded and virtually the entire early left-sided venous contrast return flowed in a retrograde fashion through the left internal jugular vein (Fig 1C). Therefore, the arterialized intracranial venous flow was attributable to retrograde flow in the left jugular vein system.

Recanalization of the left innominate vein and placement of a $12 \times 40 \mathrm{~mm}$ Wallstent (Boston Scientific, Natick, Mass) was then performed. Following recanalization, normal antegrade flow returned to the left jugular vein. Follow-up MR imaging was performed 5 months after recanalization (Fig 2). The study showed resolution of the abnormal signal intensity within the left cavernous sinus, petrosal sinus, and superior ophthalmic vein. There was some residual abnormal signal intensity present within the left transverse sinus, believed to represent persistent arterialized flow or partial thrombus formation. At the time of the follow-up scan, the patient's visual symptoms had resolved.

Received August 17, 2006; accepted after revision September 19.

From Clinical Radiologists, SC, Southern Illinois University School of Medicine, Department of Radiology, Memorial Medical Center, Springfield, III.

Please address correspondence to Ryan Watson, MD, Department of Radiology, Southern Illinois University School of Medicine, Memorial Medical Center, 701 N First St, Springfield, IL 62781; e-mail: watsonr@clinicalradiologist.com

DOI 10.3174/ajnr.A0504

\section{Discussion}

Arterialized blood flow in the cavernous sinus may be seen on MRA as increased signal intensity. The main differential diagnosis includes direct cavernous sinus carotid artery fistula, dural venous fistula, as well as cervical arteriovenous malformation and cervical dural venous fistula. ${ }^{1}$ We encountered an unusual case of arterialized blood flow in the cavernous sinus on MRA, resulting from arterialized retrograde venous flow in the internal jugular vein. This abnormal flow originated from an upper extremity dialysis arteriovenous fistula in the presence of central venous occlusion.

Normally, intracranial venous flow is not depicted on MRA because cephalocaudad venous signal intensity is saturated by placement of saturation bands over the superior sagittal sinus. Some high signal intensity in the cavernous sinus on MRA can be normal (seen in 4\%-36\% of asymptomatic patients), especially when most of the signal intensity is limited either to only the most anterior or to posterior portions of the sinus. This is believed to be related to drainage from incompletely saturated venous blood entering the cavernous sinus from the superficial middle cerebral vein or the superior petrosal sinus. ${ }^{2-4}$

Complete left brachiocephalic vein occlusion is a relatively uncommon but potentially underdiagnosed entity. ${ }^{5}$ The most frequent causes are venous catheter placement and malignancy. A study performed by Otten et $\mathrm{al}^{6}$ found that 3 patients of 9 with isolated brachiocephalic vein occlusion had no history of central lines but did have extremity AV shunts for dialysis, similar to that in our patient. None of the patients in their study revealed any central nervous system symptoms.

The presented case shows abnormal flow signal intensity in the cavernous sinus on MRA, but the usual causes were excluded. The patient's brachiocephalic vein occlusion, ipsilateral to an upper extremity AV fistula dialysis graft, diverted arterialized venous flow retrograde into the jugular vein. This arterialized retrograde venous flow was demonstrated as increased signal intensity in the dural sinuses and distended ophthalmic vein on MRA. The follow-up MR imaging performed after brachiocephalic vein recanalization shows nearly complete resolution of the abnormal dural sinus flow signal intensity, indicating greatly decreased intracranial venous pressures. At the time of the follow-up scanning, the patient reported no visual symptoms.

The presented case illustrates an additional cause for the appearance of arterialized dural sinus flow on MRA. Arterialized venous flow from an upper extremity arteriovenous dialysis fistula was diverted intracranially because of central ve- 

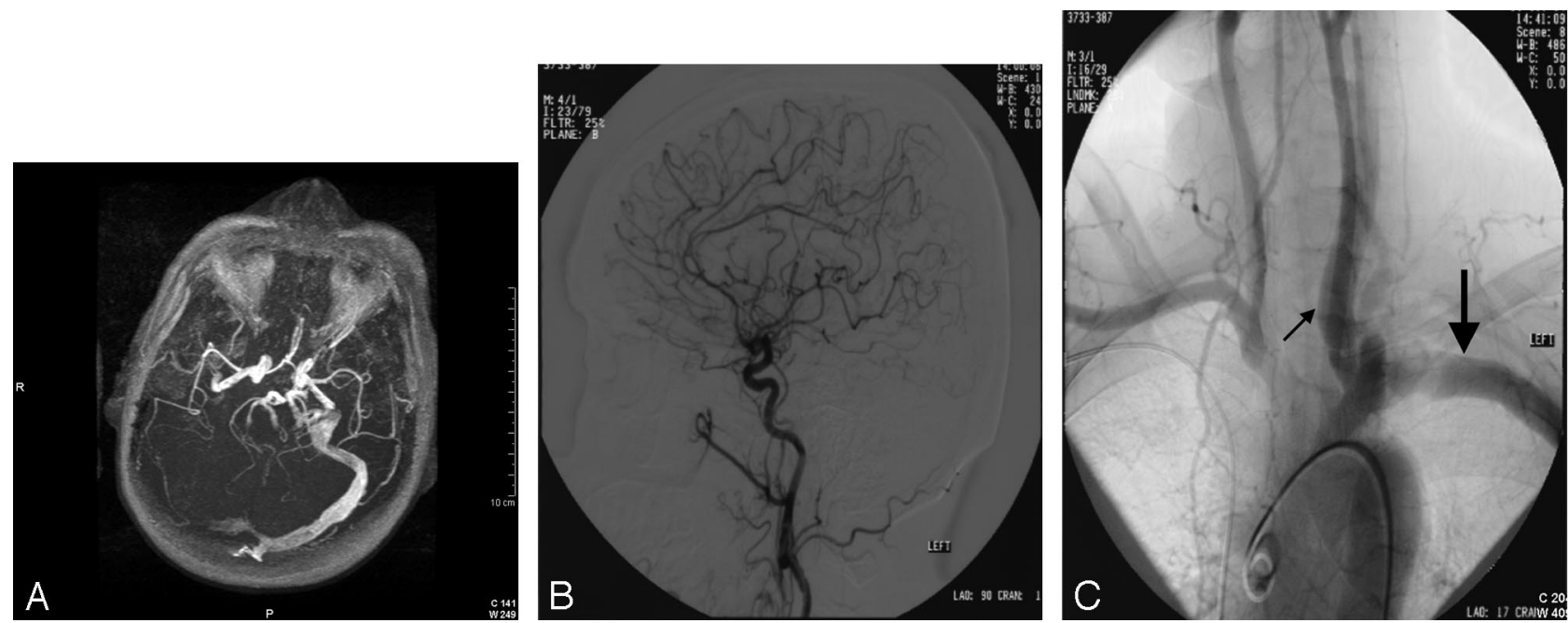

Fig 1. A, Axial MRA maximum intensity projection (MIP) (TR, 34; TE, 4) shows signal intensity within the left cavernous sinus, petrosal sinus, and transverse sinus. $B$, Digital subtraction lateral angiogram with selective injection of left internal carotid artery. There is no evidence of venous flow, but there is some reflux into the external carotid artery. $C$, Conventional angiogram, late arterial phase, shows early filling of left subclavian vein (large arrow) due to ipsilateral AV fistula and retrograde flow in the left jugular vein (with the left common carotid artery superimposed) (small arrow).

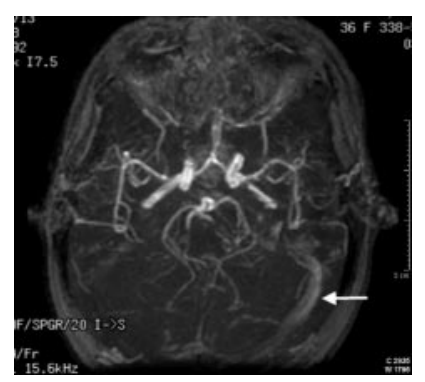

Fig 2. A 5-month follow-up axial MRA MIP (TR, 50; TE, 6.9) shows no abnormal signal intensity in the cavernous sinus or petrosal sinus. There is a small amount of increased signal intensity in the left transverse sinus (arrow).

nous occlusion. This abnormal flow was seen as abnormally increased dural sinus signal intensity on MRA, mimicking symptoms and characteristics of carotid cavernous sinus fistula.

\section{References}

1. Stark D, Bradley W, eds. Magnetic Resonance Imaging. St. Louis: C.V. Mosby; 1999:1308,1660-61

2. Tanka H, Fujita N, Hirabuki N, et al. Origin of high signal intensity in the cavernous sinus in MR angiographic source images: correlation between MR and conventional angiography. J Comput Assist Tomgr 2004;28:728-34

3. Rucker JC, Biousse V, Newman NJ. Magnetic resonance angiography source images in carotid cavernous fistulas. Br J Ophthalmol 2004;88:311

4. Ouanounou S, Tomsick TA, Heistman C, et al. Cavernous sinus and inferior petrosal sinus flow signal on three-dimensional time-of-flight MR angiography. AJNR Am J Neuroradiol 1999;20:1476-81

5. Gonsalves CF, Eschelman DJ, Sullivan KL, et al. Incidence of central vein stenosis and occlusion following upper extremity PICC and port placement. Cardiovasc Intervent Radiol 2003;26:123-27

6. Otten TR, Stein PD, Kalpesh PC, et al. Thromboembolic disease involving the superior vena cava and brachiocephalic veins. Chest 2003;123:809-12 